\title{
Estimador neuronal del ángulo del rotor de generadores sincrónicos a partir de mediciones de tensión y corriente en terminales
}

\author{
René A. Barrera*, Juan J. Mora**, Sandra M. Perez **\$ \\ *Escuela de Ingenierías Eléctrica, Electrónica y de Telecomunicaciones, \\ Universidad Industrial de Santander, Bucaramanga, Colombia \\ **Programa de Ingeniería Eléctrica, Universidad Tecnológica de Pereira, \\ Pereira, Colombia \\ §e-mail: saperez@utp.edu.co
}

\section{Resumen}

En este artículo se desarrolla un modelo tipo caja negra para la estimación del ángulo del rotor de un generador sincrónico, utilizando mediciones de tensión y corriente en los terminales. El modelo está basado en redes neuronales del tipo perceptrón multicapa (MLP). A partir de simulaciones en el dominio del tiempo de un sistema de potencia básico consistente en un generador conectado a un barraje infinito, se obtienen los registros de tensión y corriente, los cuales se utilizan como base de datos para entrenar y validar la red neuronal propuesta para la estimación del ángulo del rotor. Se encuentra que el modelo basado en redes neuronales se adapta muy bien al modelo clásico del generador, mostrando un error cuadrático medio inferior a $1 \times 10^{-10}$. Los resultados obtenidos demuestran la validez del método de estimación y promueven su uso potencial para estudios de estabilidad del generador sincrónico.

Palabras clave: Redes neuronales, Generador sincrónico, Ángulo del rotor.

ELECTRICAL ENGINEERING

\section{Neural estimator of the rotor angle of synchronous generators from the measurement of voltage and current at terminals}

\begin{abstract}
In this work, a black-box model for the estimation of the rotor angle of a synchronous generator is developed by using voltage and current measurements at the terminals. The model is based on neural networks of the multilayer perceptron (MLP) type. From time-domain simulations for a basic power system, which consists of a generator connected to an infinite bus bar, voltage and current records are collected, which are used as a database to train and validate the neural network that is proposed for the estimation of the rotor angle. It is found that the neural-network based model adapts itself very well to the classic model of the generator, exhibiting a mean square error of $1 \times 10^{-10}$. The results show the validity of the estimation method and foster its potential use for stability studies of the synchronous generator.
\end{abstract}

Keywords: Neural networks, Synchronous generator, Rotor angle. 


\section{Introducción}

Los sistemas eléctricos de potencia han tenido un gran desarrollo en los últimos años en respuesta al crecimiento de la población y a la necesidad de brindar un servicio eficiente y de mejor calidad. Los generadores sincrónicos no han sido ajenos a esta evolución ya que desempeñan un papel importante dentro de los sistemas eléctricos. Por ello, se hace necesario estudiar su comportamiento bajo operación en estado estable y transitorio, utilizando modelos apropiados para la máquina, especialmente con el fin de realizar análisis válidos de estabilidad. Casi ocho décadas después de la primera publicación en esta área, el tema aún sigue siendo una atractiva y desafiante materia de investigación (Kilgore, 1931; Wright, 1931).

Para el modelado del generador sincrónico se han empleado diversas aproximaciones y enfoques para pasar por alto principalmente la no linealidad inherente a la representación a gran escala del sistema de potencia y la sobrecarga computacional asociada a su compleja dinámica (Park et al., 2002, 2005). Entre algunas de las metodologías utilizadas, se encuentran las redes neuronales artificiales, las cuales han sido ampliamente utilizadas sobre todo en la identificación y control de sistemas no lineales. Las redes neuronales han sido ampliamente empleadas en aplicaciones del sector eléctrico y más específicamente en el modelado del generador sincrónico, demostrando que son una alternativa viable para la solución del problema en cuanto a exigencia computacional y complejidad del modelo (Chow \& Thomas, 1989; Ravan et al., 2004; Teeuwsen et al., 2003; Tsai \& Keyhani, 1995). Sin embargo, las propiedades y limitaciones fundamentales de las redes neuronales deben ser cuidadosamente examinadas para: a) determinar si el modelo de la red neuronal puede ser entrenado utilizando medidas disponibles del generador y para diferentes condiciones de operación; b) conocer cuáles deben ser los patrones de entrenamiento para el modelo de representación del generador sincrónico; c) explorar la posibilidad de adaptación del modelo basado en redes neuronales a los modelos matemáticos ya existentes y bien desarrollados del generador sincrónico para propósitos de evaluación de desempeño y valoración de la estabilidad dinámica; y d) saber si el modelado del generador, utilizando redes neuronales artificiales, tiene la capacidad de generalizar a partir de los casos de entrenamiento y también de responder con exactitud ante los casos para los cuales no ha sido entrenada (Tsai \& Keyhani, 1995).

Entre los propósitos del análisis de estabilidad en un sistema de potencia, está el de examinar el comportamiento dinámico del generador sincrónico bajo diversos tipos de perturbaciones. Dichos análisis se pueden realizar a través del estudio de simulaciones en el dominio de la frecuencia (análisis modal) o en el dominio del tiempo, las cuales requieren de complejos conjuntos de ecuaciones diferenciales no lineales que están en función de los parámetros del generador y que para resolverlos se requiere, en algunos casos, de un considerable esfuerzo computacional. En casos en los cuales no se conocen dichos parámetros o no se tiene bien definida la estructura del modelo de la máquina, se puede recurrir al modelado tipo caja negra, que sólo considera la relación entre un conjunto de datos de entrada y salida de un sistema.

El desarrollo de este tipo de modelos es importante porque debido a consideraciones económicas, la operación del sistema de potencia se restringe muy cerca de los límites de estabilidad, para lo cual se requieren de medidas confiables y exactas de las diversas variables relacionadas que permitan determinar en todo momento una operación adecuada y segura. Entre éstos, los esquemas de control de emergencia en tiempo real, constituyen el último recurso y la barrera importante de defensa contra los apagones (Pavella et al., 2000). El análisis de estabilidad transitoria del ángulo del par permite evaluar la habilidad del sistema para no perder el sincronismo ante disturbios y posibilita las acciones correctivas apropiadas, cuando así se requiera. Para monitorear y controlar la estabilidad transitoria en tiempo real, el ángulo del rotor y la velocidad de los generadores sincrónicos son las cantidades más importantes a medir. Si estas cantidades pueden ser medidas 
con suficiente rapidez y exactitud se utilizan para el monitoreo de pérdida de sincronismo en tiempo real y para diseñar esquemas automáticos de estabilización de lazo cerrado (Del Angel et al., 2007).

En este artículo se presenta un modelo tipo caja negra del generador sincrónico para la estimación del ángulo de par, ante condiciones de falla, a partir de las redes neuronales artificiales, utilizando medidas de tensión y corriente en terminales. En la Sección 2 se presentan los fundamentos básicos de las redes neuronales, aunque una explicación detallada está fuera del alcance de este artículo. La Sección 3 está dedicada al planteamiento de la metodología propuesta para la estimación del ángulo del rotor del generador sincrónico. En la Sección 4 se muestran los resultados de las pruebas realizadas a la metodología propuesta, para el caso específico de un generador conectado a una barra infinita por medio de dos líneas de transmisión en paralelo. Finalmente, en la Sección 5 se presentan las conclusiones derivadas de esta investigación.

\section{Redes neuronales perceptrón multicapa}

En esta sección se presentan brevemente algunos de los aspectos más importantes de las redes neuronales artificiales (ANN, Artificial neural networks), utilizados para el desarrollo de esta investigación. Una información más completa sobre ANN puede ser encontrada en los trabajos de Rusell \& Norvig (2002), Looney (1997) y Haykin (1994).

\subsection{Aspectos básicos}

Las ANN son caracterizadas por su arquitectura, algoritmos de entrenamiento o aprendizaje y funciones de activación. La arquitectura describe básicamente las conexiones entre las neuronas y consiste en una capa de entrada, una capa de salida y generalmente, una o varias capas ocultas entre éstas. En la Figura 1 se muestra una de las redes comúnmente usadas, conocida como red neuronal artificial de propagación hacia delante (feed forward ANN), con una capa oculta. Las capas en esta red son interconectadas por enlaces de comunicación que están asociados con pesos, los cuales establecen los efectos en la información

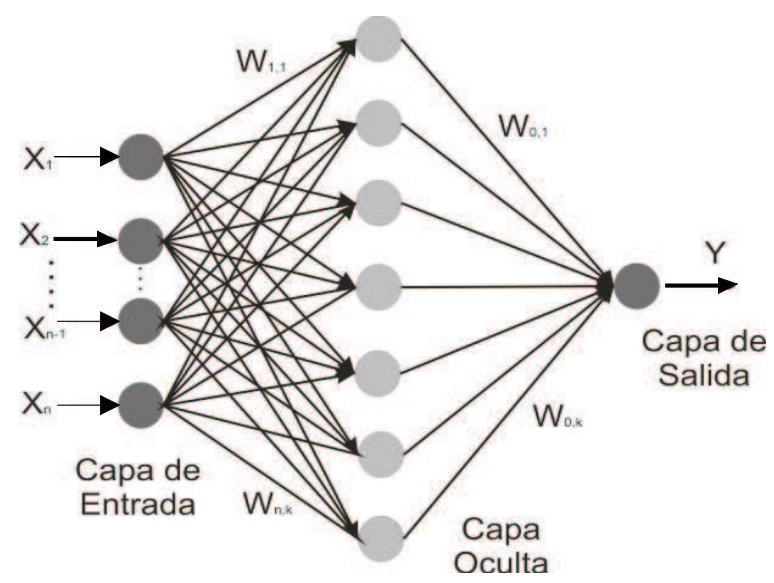

Fig. 1. Red neuronal típica con una capa oculta.

que pasa a través de ellos; estos pesos son determinados por el algoritmo de entrenamiento (Aranguren \& Muzachiodi, 2003).

Dependiendo del algoritmo de entrenamiento, las ANN pueden ser clasificadas del siguiente modo: a) redes de pesos fijos, las cuales no necesitan ningún tipo de entrenamiento; b) redes no supervisadas, que se entrenan para un ajuste de pesos en las conexiones a partir de sólo los datos de entrada; c) redes supervisadas que son las más comúnmente empleadas y que utilizan en el entrenamiento los datos de entrada y salida para actualizar los pesos y umbrales. La red tipo perceptrón multicapa (MLP, multilayer perceptron) está dentro de esta última categoría.

La función de activación relaciona la salida de una neurona ante sus entradas, a partir del nivel de actividad de cada una de las entradas de la neurona. Algunas de las funciones de activación comúnmente utilizadas son las siguientes: escalón (umbral), lineal mixta, identidad, sigmoidal y tangente hiperbólica (Haykin, 1994). El proceso de aprendizaje de la red perceptrón multicapa involucra el empleo de los datos de entrada y salida para determinar los pesos y umbrales. Una de las técnicas utilizadas para obtener estos parámetros es el algoritmo de retropropagación (back-propagation) descrito por Haykin (1994) y Aranguren \& Muzachiodi (2003). En este algoritmo, los pesos y umbrales son ajustados iterativamente para lograr un valor mínimo del error cuadrático medio entre la salida de la red y el valor deseado, tal como se presenta en la sección de pruebas y resultados. 


\subsection{Estrategia básica de ajuste del perceptrón multicapa}

El perceptrón multicapa (MLP) es la red neuronal más comúnmente utilizada en aplicaciones tales como reconocimiento de patrones, control y clasificación, entre otras (Chow \& Thomas, 1989). La estrategia básica de ajuste en la mayoría de aplicaciones reportadas y que se aplica en esta investigación consta de los siguientes pasos:

Paso 1. Selección de las entradas y extracción de las características. Este es el primer paso para cualquier problema de reconocimiento de patrones y consiste básicamente en la determinación de cuáles de las variables disponibles (posibles entradas), tienen alguna relación con lo que se desea identificar como salida. Este paso es importante, ya que define tanto el desempeño como el tamaño de la ANN.

Paso 2. Definición de los datos de entrenamiento. La definición del conjunto de datos de entrenamiento es importante, ya que éstos deben contener información sobre todas las salidas posibles que se desean identificar. Los datos de entrenamiento ayudan a determinar los parámetros de la red.

Paso 3. Selección de la ANN. En este paso se define el tamaño y arquitectura de la red (número de neuronas, entradas, capas ocultas, neuronas ocultas, etc.). Este paso se puede realizar antes de iniciar el ajuste de la red, aunque recientemente se han propuesto otras metodologías que permiten variaciones en la arquitectura en función de un mejor desempeño (Mora-Flórez et al., 2006).

Paso 4. Entrenamiento de la ANN. En este proceso, a partir de la presentación de datos de entrada (variables) y de datos de salida (clases a reconocer), tomados del conjunto de entrenamiento, se establece una relación entre entradas y salidas. Esta relación es del tipo caja negra, es decir que ésta no se puede presentar de manera explicita, lo que constituye una de las desventajas de las redes neuronales. En el entrenamiento, comúnmente los pesos y umbrales de la red son actualizados solamente después que la totalidad del conjunto de datos ha sido aplicado a la red.
Paso 5. Prueba de la red neuronal. Utilizando datos diferentes a los que se emplearon en el entrenamiento, se puede obtener un índice de confianza en la red neuronal. Uno de los índices más utilizados es el que se presenta a continuación y que está dado para el caso en el cual la salida puede tomar muchos valores o clases y es de naturaleza discreta:

$$
\text { Confianza }=\frac{\begin{array}{l}
\text { Datos de } \\
\text { prueba }
\end{array}-\begin{array}{l}
\text { Datos bien } \\
\text { clasificados }
\end{array}}{\begin{array}{l}
\text { Datos de } \\
\text { prueba }
\end{array}}
$$

Para el caso donde hay una sola salida, se utiliza como índice de medición el error medio cuadrático $E$ entre la salida deseada y la obtenida. Este índice es el que se utiliza en esta aplicación, así:

$$
E=\sqrt{\sum_{i=1}^{N} \frac{\left(\delta_{i-\text { real }}-\delta_{i-\text { estimado }}\right)^{2}}{N}}
$$

donde $N$ es el número total de muestras comparadas, y $\delta$ corresponde al ángulo del rotor de la máquina (real y estimado por la $\mathrm{ANN}$ ).

\section{Desarrollo de una red neuronal para estimar el ángulo del rotor}

El propósito de la ANN planteada es la estimación del ángulo del rotor del generador sincrónico, empleando la tensión y corriente medidas en terminales. En este numeral se presentan los aspectos más importantes para el ajuste de la red neuronal.

\subsection{Selección de las entradas}

Como entradas de la red neuronal se seleccionaron la tensión, la corriente y el ángulo de la tensión y de la corriente, medidos en los terminales de la máquina en los instantes $t, t-1$ y $t-2$. La salida del modelo basado en la red neuronal consiste en una neurona que representa el ángulo del rotor para un punto de operación específico, así: 


$$
\delta(t)=f\left\{\begin{array}{l}
v(t), v(t-1), v(t-2), i(t), i(t-1), i(t-2), \\
\theta_{v}(t) \theta_{v}(t-1), \theta_{v}(t-2), \theta_{i}(t), \theta_{i}(t-1), \theta_{i}(t-2)
\end{array}\right\}
$$

donde $v(t)$ e $i(t)$ son la tensión y corriente en terminales para el tiempo $t$. De otra parte, $v(t-1)$, $v(t-2), i(t-1), i(t-2)$ corresponden al fundamental de la tensión y corriente en el instante $t-1$ y $t-2$, respectivamente. Finalmente, $\theta_{v}$ y $\theta_{i}$ representan el ángulo de la tensión y de la corriente, respectivamente, en los mismos instantes de tiempo. En total, se seleccionaron 12 entradas para obtener una salida que corresponde al ángulo del rotor de la máquina $(\delta)$.

\subsection{Selección de la ANN y determinación de la arquitectura}

La red neuronal utilizada es del tipo perceptrón multicapa de propagación hacia adelante y con una capa oculta. Las funciones de activación utilizadas son la tangente hiperbólica (tansig, en MATLAB ${ }^{\circledR}$ ), y lineal pura (purelin). La función purelin realiza un mapeo de la entrada a través de una línea recta de pendiente unitaria, así:

$$
\operatorname{purelin}(x)=x
$$

Por otro lado, la función tansig representa una función tangente hiperbólica, que es una función continua no lineal con un rango comprendido entre -1 y 1 , así:

$$
\operatorname{tansig}(x)=\frac{2}{1+e^{-2 x}}-1
$$

La función de activación para las neuronas de la capa oculta es la tangente hiperbólica y para las neuronas de salida es la función lineal. En la Figura 2 se presenta la red empleada en este trabajo.

El número de neuronas en la capa oculta se determinó experimentalmente de acuerdo al estudio del comportamiento de la red durante el proceso de entrenamiento, teniendo en cuenta algunos factores como la velocidad de

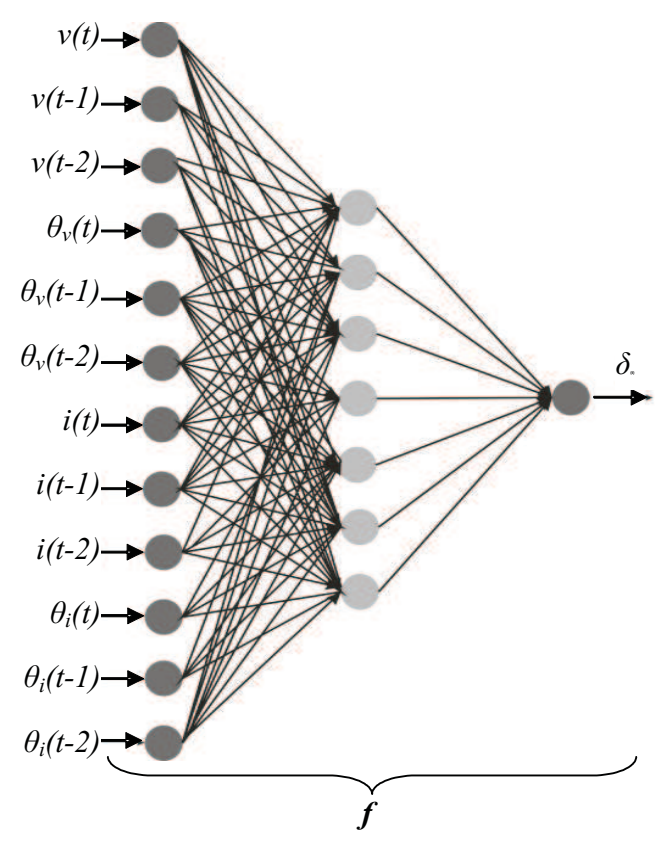

Figura 2. Red neuronal artificial propuesta para la estimación del ángulo del rotor.

convergencia y el criterio de error, entre los más importantes.

La Figura 3 muestra la variación del número de neuronas en la capa oculta y el error cuadrático medio de la red neuronal al final del entrenamiento. Dentro de los parámetros del algoritmo de entrenamiento se encuentra el error cuadrático medio de meta y el número máximo de épocas. Para esta prueba se asigna una meta muy baja $\left(1 \times 10^{-20}\right)$, con el fin de que el algoritmo no converja rápido y se aprecie el óptimo desempeño de la red para un número máximo de 5,000 épocas de aprendizaje.

En la Figura 4 se muestra la variación de la velocidad de convergencia de la red (medida en épocas) respecto al número de neuronas presentes en la capa oculta. Para observar la velocidad de convergencia, se plantea un esquema de 


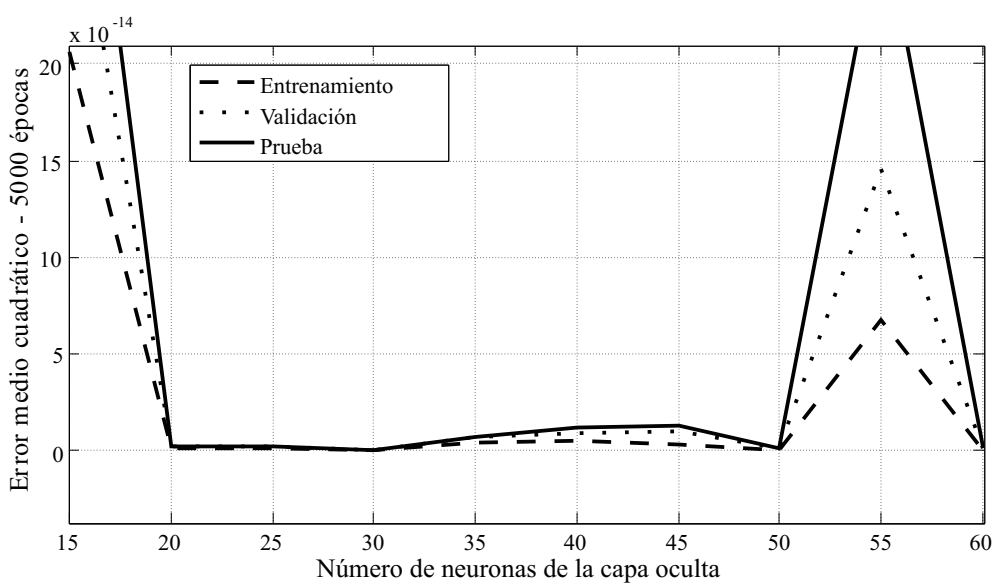

Figura 3. Desempeño de la red (error cuadrático medio de meta de $10^{-20}$ y número máximo de épocas de 5,000).

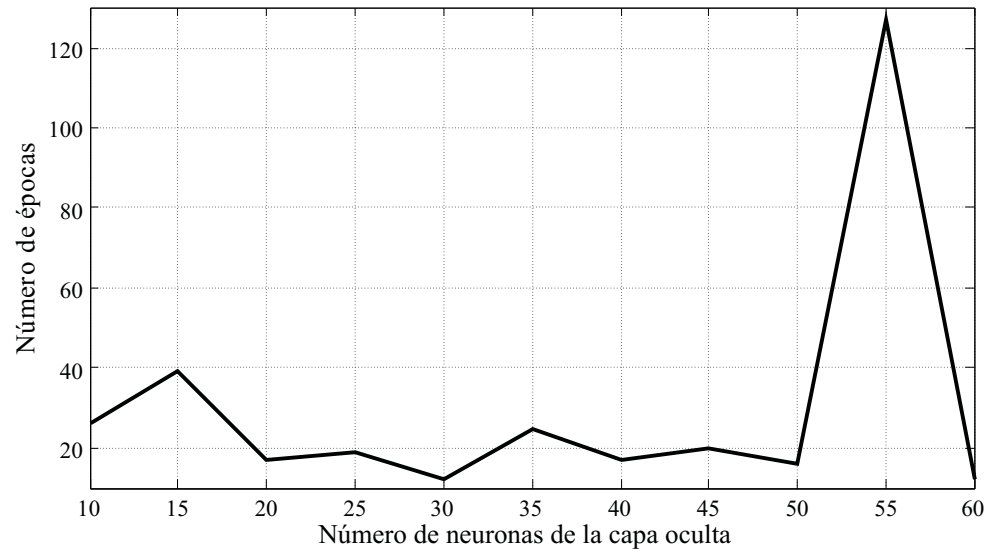

Figura 4. Velocidad de convergencia de la red con un error cuadrático de meta de $10^{-10}$.

entrenamiento con una meta de error no tan exigente, pero aceptable para este caso $\left(1 \times 10^{-10}\right)$ y se centra la atención en el número de épocas necesario para lograr la meta de error.

Con base en esa información, el número de neuronas utilizado es de 30 , puesto que presenta una convergencia apropiada y un error cuadrático medio global más bajo. Adicionalmente, se observa que para 55 neuronas de la capa oculta se obtienen resultados muy desfavorables, lo que prueba que el aumento de neuronas no siempre mejora el rendimiento global de la red neuronal artificial, pero sí aumenta el esfuerzo computacional.

\section{Pruebas y resultados}

\subsection{Sistema de prueba}

La red neuronal propuesta se prueba en el sistema de potencia presentado en la Figura 5. El sistema consiste en una estación de generación compuesta por 4 máquinas de $555 \mathrm{MVA}, 24 \mathrm{kV}$ y $60 \mathrm{~Hz}$, que suple potencia a una barra infinita conectada a través de dos líneas de transmisión. Esta configuración simple es muy útil para la comprensión de conceptos y efectos básicos en estabilidad transitoria (Kundur, 1994; Anderson \& Fouad, 1997). 


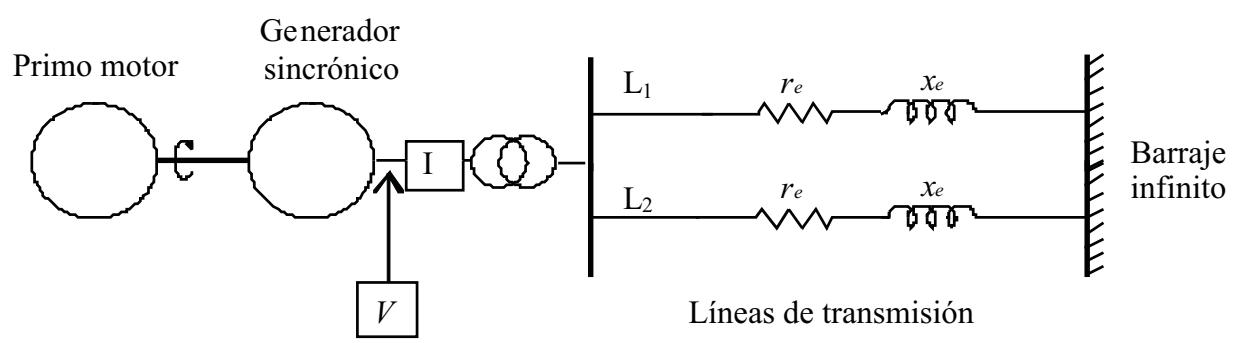

Figura 5. Sistema de un generador sincrónico conectado a una barra infinita.

Los cuatro generadores se modelan como un único generador equivalente representado por el modelo clásico con los parámetros expresados por unidad con la base de 2,220 MVA (4×555), $24 \mathrm{kV}$ y $60 \mathrm{~Hz}$, con una constante de inercia $(H)$ de $3.5 \mathrm{MW} \times \mathrm{s} / \mathrm{MVA}$, coeficiente de amortiguamiento $\left(K_{D}\right)$ igual a cero y la reactancia transitoria $\left(X_{d}\right)$ de 0.3 p.u. No se consideran los efectos del gobernador de velocidad.

Los valores de las reactancias del transformador y de las líneas de transmisión están dados por unidad en la misma base y referidas al lado de baja tensión del transformador, así: la reactancia del transformador es de 0.15 y las reactancias de las líneas 1 y 2 son iguales a 0.50 y 0.93 , respectivamente. Los valores de las resistencias se asumen muy pequeños por lo cual no se consideran significativos.

El punto de operación inicial del generador expresado por unidad está dado por $P=0.9$, $Q=0.436$ (sobreexcitado), $V_{\mathrm{t}}=1.0\left\llcorner 28.34^{\circ}\right.$, $V_{\text {inf }}=0.90081\left\llcorner 0^{\circ}\right.$.

Para el desarrollo del estimador del ángulo se utilizó el módulo de redes neuronales de MATLAB $®$ (Demuth et al., 2006). Los pesos y los umbrales iniciales son valores aleatorios entre 0 y 1 . Las entradas son normalizadas a valores entre -1 y 1 .

\subsection{Pruebas realizadas}

Un sistema de potencia puede estar sujeto a diferentes clases de disturbios, por lo que resulta complejo utilizar todas las respuestas del sistema ante diferentes disturbios en el conjunto de entrenamiento. Para el análisis aquí presentado, se considera el sistema sujeto a cortocircuitos trifásicos en la línea de transmisión identificada como $\mathrm{L}_{2}$. Todas las fallas trifásicas aparecen a los 0.1 seg de simulación y se despejan sacando de servicio la línea que falla, en diferentes instantes de tiempo, para conseguir varias duraciones de la falla. Esta es una práctica común en estudios de estabilidad. Todos los disturbios fueron aplicados para el sistema en la misma condición de operación (aproximadamente 2,000 MW).

En total, se simularon 15 eventos para el ajuste y prueba de la red. Un evento se define como cada simulación de una falla trifásica y su eliminación en un instante de tiempo del intervalo comprendido entre $10 \mathrm{~ms}$ y $120 \mathrm{~ms}$. De cada evento se obtienen aproximadamente 1,000 pares de entradas / salidas, conformados por las muestras de la señal y que son necesarios para entrenar la red neuronal. Según lo anterior, la información que ingresa a la red consta de datos de las tensiones y corrientes en terminales y del ángulo del rotor del generador para cada instante de tiempo. Para el conjunto de entrenamiento se toma el $80 \%$ de los datos disponibles, o sea que se seleccionan aleatoriamente 12 de los 15 eventos simulados. Como datos de prueba para la red neuronal se utilizan $3(20 \%)$ eventos diferentes a los de entrenamiento. Adicionalmente, de los datos de entrenamiento, se toman aleatoriamente dos eventos para ingresarlos al algoritmo de entrenamiento como datos de validación.

En la Figura 6 se presenta el desempeño de la red neuronal empleada para la estimación del ángulo de rotor. Se observa que el error cuadrático medio en la etapa de entrenamiento y prueba siempre fue menor a $1 \times 10^{-10}$. 


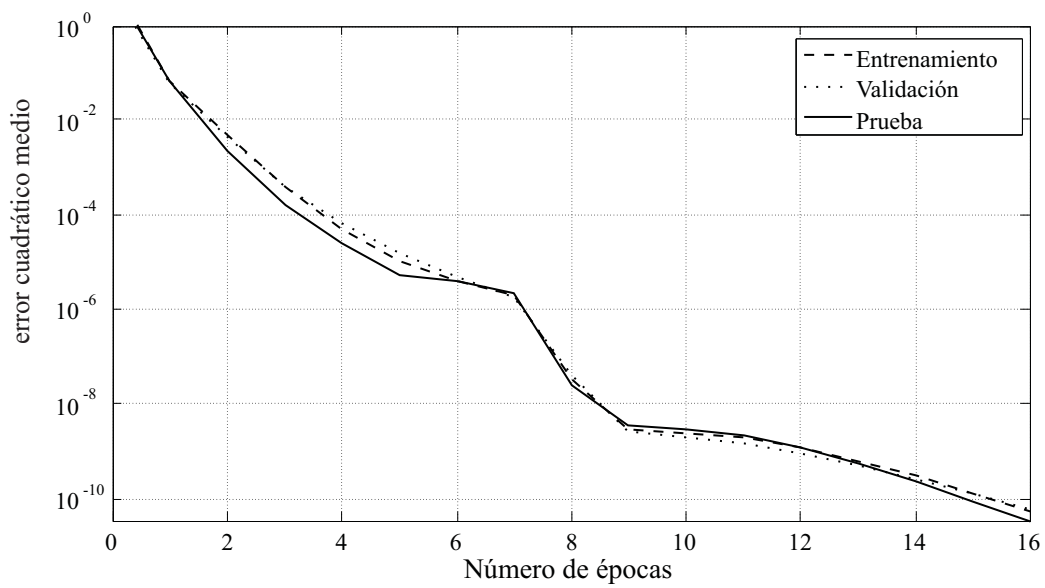

Figura 6. Desempeño de la ANN (medido por el error cuadrático medio) en función del número de épocas de entrenamiento.

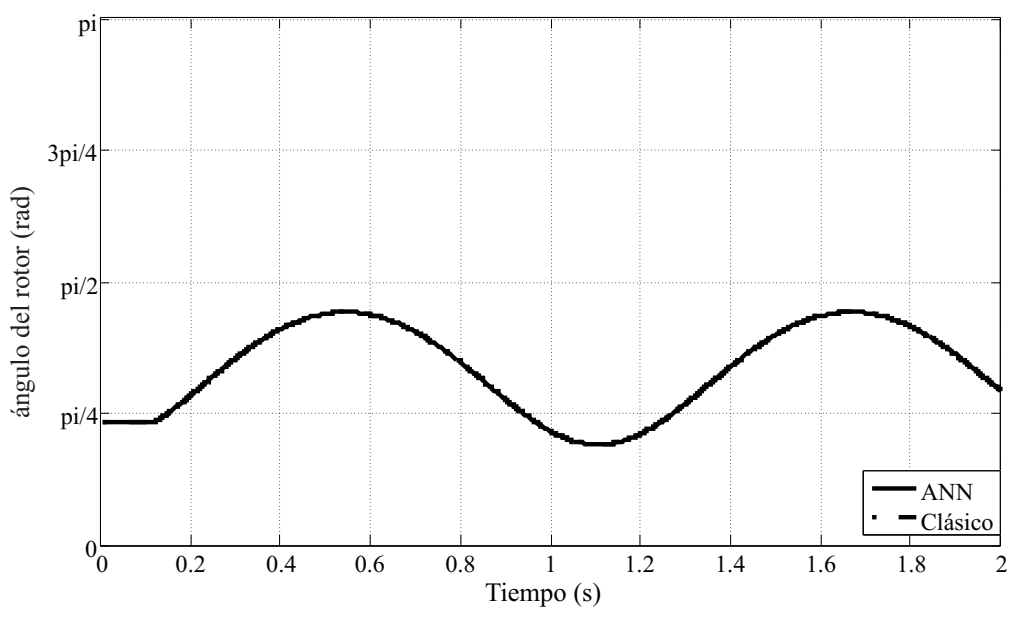

Figura 7. Comparación de los estimadores del ángulo del rotor ante un caso estable.

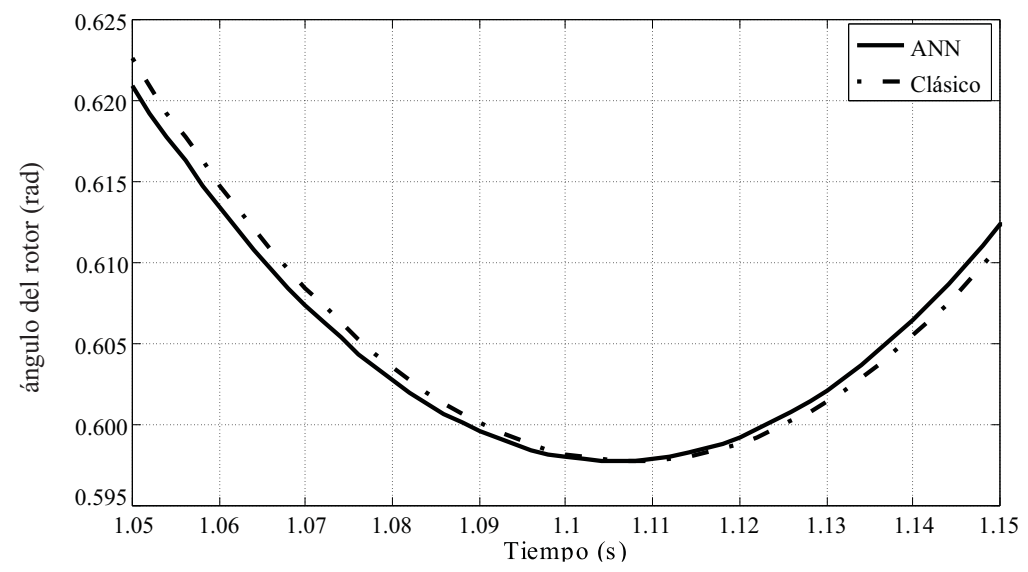

Figura 8. Detalle de la comparación de los estimadores del ángulo del rotor ante un caso estable. 
El conjunto de datos para entrenamiento y validación de la red neuronal se obtienen por medio de simulación a partir del modelo clásico del generador sincrónico presentado en el Apéndice A. El intervalo de tiempo para las simulaciones es de 2 seg para el caso estable o críticamente estable, mientras que para el caso inestable la simulación se detiene cuando el ángulo llega a $180^{\circ}$ (situación que presenta un serio problema de inestabilidad). La duración de 2 seg para la simulación se elige por inspección de la respuesta de la máquina (la onda resultante presenta completamente su tendencia en todos los casos antes de los $2 \mathrm{seg}$ ). La frecuencia de muestreo utilizada en las pruebas es de $500 \mathrm{~Hz}$.

\subsection{Análisis de resultados}

En las Figuras 7, 9 y 11 se muestran los resultados obtenidos en la estimación del ángulo del rotor para un generador sincrónico, utilizando un estimador neuronal y considerando tres situaciones de estabilidad (estable, críticamente estable e inestable). Estos resultados se comparan con los valores obtenidos con el modelo clásico del generador sincrónico (que para este estudio representa la máquina real). Para facilitar la comparación, en estas figuras se representa en la misma escala el ángulo del rotor. El tiempo de despeje crítico (TDC) para el sistema de potencia en el punto de operación analizado es de $0.087 \mathrm{seg}$. En general, para cualquier generador sincrónico, si el tiempo de despeje es mucho menor que este valor, la respuesta del sistema siempre es estable.

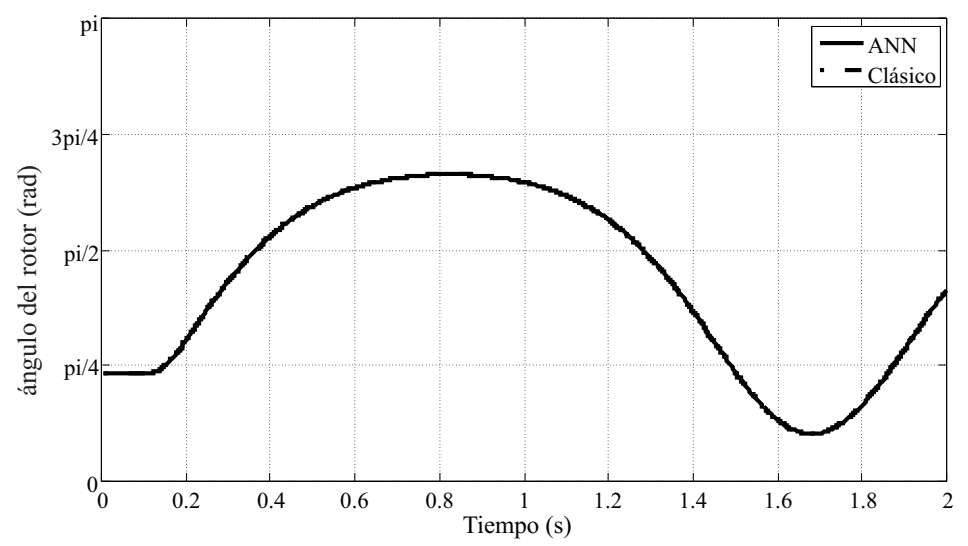

Figura 9. Comparación de los estimadores del ángulo del rotor ante un caso críticamente estable.

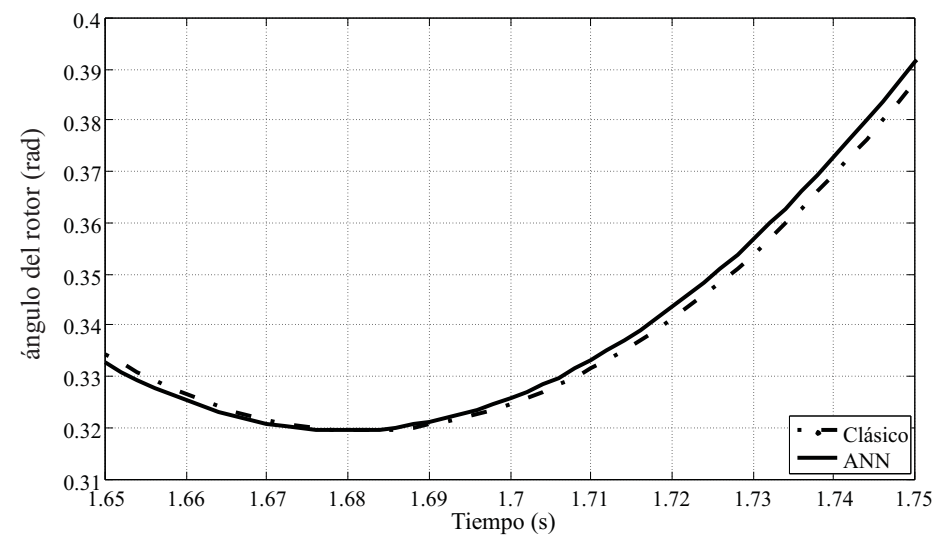

Figura 10. Detalle de la comparación de los estimadores del ángulo del rotor ante un caso criticamente estable. 


\subsubsection{Caso estable (tiempo de despeje menor que el valor crítico)}

La evolución del ángulo del rotor (el obtenido a partir del modelo clásico y el estimado por la ANN) para el caso estable con un tiempo de despeje de $0.04 \mathrm{seg}$ se ilustra en la Figura 7. En este gráfico no se percibe diferencia considerable entre el valor estimado y el obtenido con el modelo clásico, mostrándose la buena adaptación de la ANN propuesta. En la Figura 8 se muestra como detalle de la Figura 7, una ventana de sólo $0.1 \mathrm{seg}$ en un punto de inflexión, para evidenciar cómo el algoritmo propuesto con base en ANN sigue al desarrollado con el método clásico, con un error muy pequeño.

\subsubsection{Caso críticamente estable (tiempo de despeje similar al valor crítico)}

Cuando la duración de la falla es muy cercana al TDC, la respuesta del sistema es críticamente estable. En la Figura 9 se presenta el ángulo del rotor considerando un tiempo de despeje de 0.085 seg. De igual manera que para el caso anterior, también se presenta un detalle de duración igual a $0.1 \mathrm{seg}$ y en un punto de inflexión de la gráfica, con el fin de observar cómo el algoritmo propuesto $\mathrm{y}$ basado en ANN sigue el basado en un método clásico, tal como se muestra en la Figura 10.

\subsubsection{Caso inestable (tiempo de despeje mayor al valor crítico)}

El caso de una respuesta inestable del sistema (duración de la falla mucho mayor que el TDC), se presenta en la Figura 11. Se muestra cómo el valor del ángulo del rotor crece ante una duración de la falla de 0.92 seg hasta pasar por el valor de $\pi \mathrm{rad}$ a los $0.978 \mathrm{seg}$. En la Figura $12 \mathrm{se} \mathrm{presenta} \mathrm{un}$ detalle de duración igual a $0.1 \mathrm{seg}$ de la Figura 9, con el fin de observar cómo el algoritmo propuesto a partir de las ANN sigue el basado en un método clásico, tal como se había comprobado para los casos anteriores.

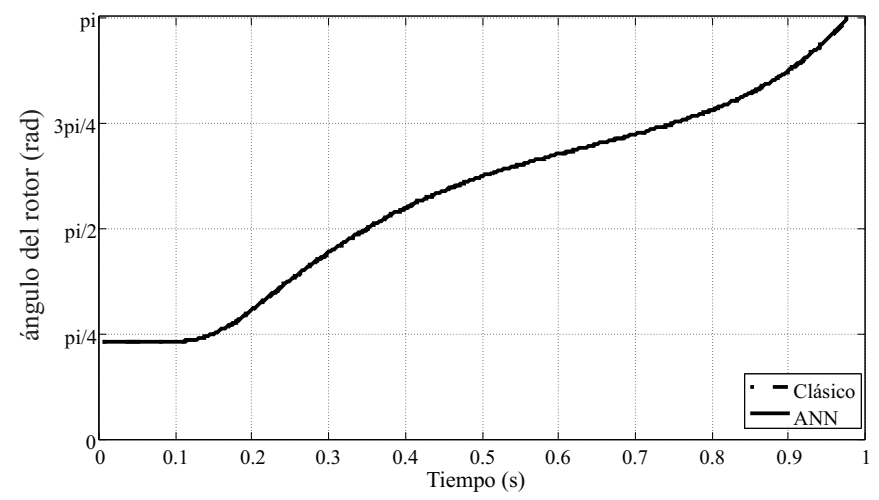

Figura 11. Comparación de los estimadores del ángulo del rotor ante un caso inestable.

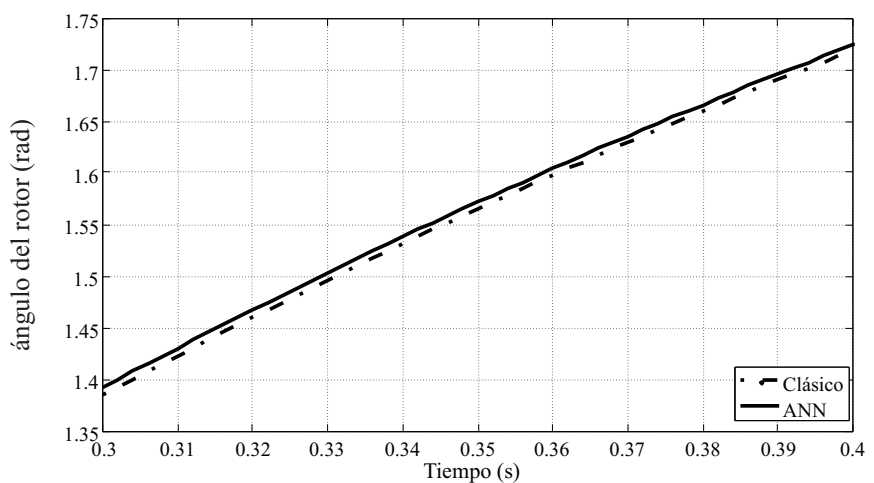

Figura 12. Detalle de la comparación de los estimadores del ángulo del rotor ante un caso inestable. 


\section{Conclusiones}

En este trabajo se presentó la aplicación de una red neuronal artificial (ANN) para la estimación del ángulo del rotor de un generador sincrónico. Los resultados obtenidos con base en simulaciones realizadas con el modelo clásico para un sistema de potencia con una sola máquina conectada a una barra infinita, fueron presentados y replicados con la red neuronal propuesta. Este modelo es fácilmente expandible y aplicable a sistemas reales considerando que se cuenta con las medidas que se requieren como entrada y también por el alto desempeño obtenido en el seguimiento de ángulo del rotor.

Finalmente, con la metodología propuesta, se presenta una alternativa sencilla para la estimación del ángulo del rotor empleando sólo las medidas fasoriales de tensión y corriente en los terminales de la máquina. Así, se propone una metodología viable para la implementación de la red neuronal entrenada con datos de simulación y basada en modelos más elaborados del generador sincrónico.

\section{Apéndice A. Modelo clásico del generador sincrónico}

En el caso de estudios en los cuales el período de análisis es pequeño en comparación con la constante de tiempo transitoria de circuito abierto, se simplifica frecuentemente el modelo del generador sincrónico asumiendo $E^{\prime} q$ constante, a través de todo el periodo de estudio. Esta suposición elimina solamente la ecuación diferencial asociada con las características eléctricas del generador, lo cual deja sólo la ecuación diferencial de movimiento asociada a la parte mecánica (ángulo del rotor del generador), así:

$$
\frac{2 H}{w_{0}} \frac{d^{2} \delta}{d t^{2}}=P_{m}-P_{\max } \operatorname{sen}(\delta)
$$

donde $P_{m}$ es la potencia mecánica de entrada en p.u., $P_{\max }$ es la potencia eléctrica máxima de salida, en p.u., $H$ es la constante de inercia en
$\mathrm{MW} \times \mathrm{s} / \mathrm{MVA}, \delta$ es el ángulo del rotor en radianes y $t$ corresponde al tiempo en seg.

Otra aproximación común simplifica el modelo del generador significativamente al ignorar el efecto transitorio de los polos salientes asumiendo que $X_{d}^{\prime}=X_{q}^{\prime}$, y también considerar que el flujo ligado al circuito de amortiguamiento permanece constante (asociado con el eje $q$ del circuito del rotor correspondiente a $X_{q}^{\prime}$ ). Con estas suposiciones (Kundur, 1994), la tensión antes de la impedancia transitoria $\left(R_{a}+j X_{d}^{\prime}\right)$ del circuito de la Figura A1 tiene magnitud constante. Por ende, la parte eléctrica del generador sincrónico queda descrita así:

$$
E^{\prime}=E_{t}+\left(R_{a}+j X_{d}^{\prime}\right) I_{t}
$$

donde $E_{t}$ corresponde a la tensión en terminales del generador, $I_{t}$ es la corriente en terminales, $E^{\prime}$ es la tensión interna inducida, $R_{a}$ es la resistencia interna del generador, que generalmente se desprecia, y $X_{d}^{\prime}$ es la reactancia transitoria de circuito abierto.

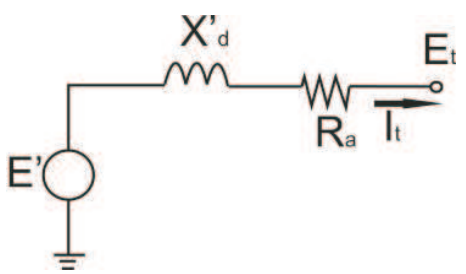

Figura A1. Circuito simplificado del modelo transitorio clásico del generador sincrónico.

\section{Referencias bibliográficas}

Anderson, P. M., \& Fouad, A. A. (1997). Power system control and stability. First Edition, Ames, Iowa: Iowa State University Press.

Aranguren, S. M., \& Muzachiodi, S. L. (2003). Redes neuronales y algoritmos genéticos. Capitulo 8: Implicancias del data mining. Tesis de grado en Sistemas de Información. Convenio UTN - ISIPER. 
Chow, M.-Y., \& Thomas, R. J. (1989). Neural network synchronous machine modeling. In Proceedings of the IEEE International Symposium on Circuits and Systems, Portland, Oregon, Vol.1, p. 495-498.

Del Angel, A., Geurts, P., Ernst, D., Glavic, M., \& Wehenkel, L. (2007). Estimation of rotor angles of synchronous machines using artificial neural networks and local PMU-based quantities. Neurocomputing 70 (16-18), 2668-2678.

Demuth, H., Beale, M., \& Hagan, M. (2006). Neural networks toolbox for use with MATLAB. User's guide, Vol. 5, The MathWorks Inc.

Haykin, S. (1994). Neural networks: a comprehensive foundation. IEEE Press, New York.

Kilgore, L. A. (1931). Calculation of synchronous machine constants. AIEE Transactions 50, 1201-1214.

Kundur, P. (1994). Power system stability and control. First edition, McGraw- Hill, Inc.

Looney, C. G. (1997). Pattern recognition using neural networks: theory and algorithms for engineers and scientists. Oxford University Press, New York.

Mora-Flórez, J.J., Pérez-Hernández, L., \& Pérez-Londoño, S.M. (2006). Utilización de redes ANFIS y señales de corriente para localización de la zona de falla en sistemas de distribución de energía eléctrica. Ingeniería e Investigación 26(3), 93-99.

Park, J.-W., Harley, R.G., \& Venayagamoorthy, G. K. (2002). Comparison of MLP and RBF neuralnetworks using deviation signals for on-line identification of a synchronous generator. In Proceedings of the 2002 IEEE Power Engineering Society Winter Meeting, Vol. 1, p. 274-279.
Park, J.-W., Venayagamoorthy, G. K., \& Harley, R. G. (2005). MLP / RBF neural-networks-based online global model identification of synchronous generator. IEEE Transactions on Industrial Electronics 52 (6), 1685-1695.

Pavella, M., Ernst, D., \& Ruiz-Vega, D. (2000). Transient stability of power systems: a unified approach to assessment and control. Kluwer Academic Publishers Group, Dordrecht, The Netherlands.

Ravan, M., Dallirrooy-Fard, R., \& Karrari, M. (2004). Synchronous generator black-box identification using continuous wavelet networks. In Proceedings of the Sixth Bulk Power System Dynamics and Control Conference, Cortina d'Ampezzo, Italy, p. 216-219.

Rusell, S. J., \& Norvig, P. (2002). Artificial intelligence: a modern approach. Second Edition, Upper Saddle River, New Jersey: Prentice Hall.

Teeuwsen, S.P., Erlich, I., \& El-Sharkawi, M. A. (2003). Advanced method for small-signal stability assessment based on neuronal networks. In Proceedings of the ISAP Conference, Lemnos, Greece, p. 1-6.

Tsai, H., \& Keyhani, A. (1995). Development of a neural network based saturation model for synchronous generator analys is . IEEE Transactions on Energy Conversion $10(4), 617-624$.

Wright, S.H. (1931). Determination of synchronous machine constants by test. AIEE Transactions 50, 1331-1351. 